\title{
Fatal breathing problems are four times as likely in intensive care as in general anaesthesia for surgery
}

The title of this News article (BMJ 2011;342:d2015, doi:10. $1136 / \mathrm{bmj} . \mathrm{d} 2015$ ) gave the wrong impression of the number of fatal airway problems after sedation in intensive care compared with the outcome after airway problems during anaesthesia for surgery. Instead, the headline should have read:
"Avoidable airway problems in intensive care identified by major UK study."

Cite this as: BMJ 2011;342:d2373 